\title{
Analysis of Insulating Material of XLPE Cables considering Innovative Patterns of Partial Discharges
}

\author{
Fernando Figueroa Godoy, ${ }^{1}$ Jacinto Torres Jimenez, ${ }^{1}$ Rubén Jaramillo Vacio, ${ }^{2}$ \\ Javier Yáñez Mendiola, ${ }^{3}$ and José Ángel Colin ${ }^{1}$ \\ ${ }^{1}$ Instituto Tecnológico Superior de Irapuato (ITESI), Irapuato, GTO, Mexico \\ ${ }^{2}$ Comisión Federal de Electricidad, Laboratorio de Pruebas a Equipos y Materiales (LAPEM), Irapuato, GTO, Mexico \\ ${ }^{3}$ Centro de Innovación Aplicada en Tecnologías Competitivas (CIATEC), León, GTO, Mexico
}

Correspondence should be addressed to Fernando Figueroa Godoy; fer-figueroa@hotmail.com

Received 9 March 2017; Revised 15 June 2017; Accepted 12 September 2017; Published 8 November 2017

Academic Editor: Huanqing Wang

Copyright (C) 2017 Fernando Figueroa Godoy et al. This is an open access article distributed under the Creative Commons Attribution License, which permits unrestricted use, distribution, and reproduction in any medium, provided the original work is properly cited.

\begin{abstract}
This paper aims to analyze the quality of insulation in high voltage underground cables XLPE using a prototype which classifies the following usual types of patterns of partial discharge (PD): (1) internal PD, (2) superficial PD, (3) corona discharge in air, and (4) corona discharge in oil, in addition to considering two new PD patterns: (1) false contact and (2) floating ground. The tests and measurements to obtain the patterns and study cases of partial discharges were performed at the Testing Laboratory Equipment and Materials (LEPEM) of the Federal Electricity Commission of Mexico (CFE) using a measuring equipment LDIC and norm IEC60270. To classify the six patterns of partial discharges mentioned above a Probabilistic Neural Network Bayesian Modified (PNNBM) method having the feature of using a large amount of data will be used and it is not saturated. In addition, PNN converges, always finding a solution in a short period of time with low computational cost. The insulation of two high voltage cables with different characteristics was analyzed. The test results allow us to conclude which wire has better insulation.
\end{abstract}

\section{Introduction}

The analysis of partial discharges (PDs) is important to determining the quality of insulation in high voltage equipment. When the electrical insulation system is flawed by design flaws, resulting from the manufacturing process, by mechanical damage of products when manipulated or stored, or by dryness caused by insulation aging, frequently small discharges occur; this phenomenon ends by affecting the solid insulation. The deterioration of the insulation can be aggravated over time causing it to be completely destroyed.

A novel technique is proposed for the measurement of PDs in transformers using the bandwidth for the PDs and [1] trying to reduce the noise in the measurements.

Partial discharges (PDs) are an event of partial deterioration that occurs, for example, on the surface or inside the insulation of electrical products possibly due to defects in the insulation structure. Due to the importance of the PDs several authors have presented studies to classify different types of patterns of PDs through prototypes that characterize the phenomena of PDs: superficial partial discharge and internal and crown PD $[1,2]$.

There are four usual types of PDs reported until now: (1) the first one is an internal PD in insulation material with different types of cavities [3,4]; (2) the corona discharge is a $\mathrm{PD}$ produced by sharp edges $[4,5]$; (3) electrical arborescence is generated by the combination of the corona discharge and superficial PD; (4) superficial PDs occur when there is effort on the surface of the dielectric, and usually they are presented in caps, cable ends, and the overheating of the generator windings and if a discharge reaches the surface from the outside $[4,5]$.

Insulation generates different internal cavities reproduction and different diameters of the cavity to produce internal PDs [2]. 
It produces internal DPs using a filtering system that can introduce errors at the moment of classification and the DPs transform them into energy for the interpretation and evaluation [1].

They perform different types of internal and external patterns: corona in oil, corona in air, superficial, and internal, but the results are not validated with actual measurements [3].

Various types of methods and techniques have been proposed to classify PDs patterns. The classification method depends on the type of measurement; the most common is to use the magnitude, angle, and repetition with statistic method. Subsequently extraction methods are used: Statistical Methods [2, 3, 6-8], Neural Networks [2, 3, 7, 9], Hybrids [6], and Diffuse Logic [6].

The aforementioned methods have been applied to classify the types of DPs in transformers, generators, and high voltage cables.

When it is a requirement to expand the transmission network and/or to replace sections of underground lines, it is necessary that the insulation of the cable be installed in compliance with the international quality standards in order to avoid interruptions of the electrical service due to failures in the insulation, improving the reliability, continuity, and safety of electric service.

Currently in systems of underground electric energy transmission, partial discharges in cables occur when a bad cable extrusion is carried out with insulation generating bubbles (internal PD), in the improper installation when the cable is hit or when it does not have a good insulation (external PD) or when the couplings are poorly placed with cables and left edges (corona discharge, floating ground, and poor contact) [9-11]. PD is analyzed using it as energy for XLPE cables, only studying the patterns behavior of internal PD using different numbers of cavities [10]. In reference [11] the authors present a type of sensor to determine the characteristics of PD using a prototype to classify (1) internal $\mathrm{PD}$, superficial PD, corona in air, and corona in oil.

In this case in the present research work the quality of insulation in high voltage underground XLPE cables from different manufacturers was tested. The construction of a prototype is proposed to obtain the following patterns of partial discharges: (1) internal PD, (2) superficial PD, (3) corona discharge in air, and (4) corona discharge in oil, additionally considering two new PD patterns: (1) false contact and (2) floating ground. The validation of the proposed prototype was performed using the statistical method and the norm IEC 60270 can know insulation degradation unlike acoustic measurements which allow knowing only the location of the $\mathrm{PD}$ in tests in the laboratory [12-15].

To classify the six partial discharges a Modified Bayesian Probabilistic Neural Net (PNNBM) was trained that has the characteristic of using a large amount of data and is not saturated, besides always finding a solution in a short period of time with low computational cost.

The methodology was implemented in two different manufactures of XLPE cables which is able to determine the quality of the insulation in an acceptable way.

\section{Prototype Design}

In the test laboratory, LAPEM, a prototype was developed for measurements (Figure 1), in which one could extract different types of DPs where the data will be free of noise, in order to get "clean" patterns in corona discharge, false contact, corona oil, and floating ground as well as internal and external PDs. Also this prototype was useful in also obtaining combined data from these measurements, creating a database to characterize in a better way the artificial neural network; the measuring equipment gives to us four columns, the first determines the cycle, the second the angle where the PD occurred, the third presents the electric field in $\mathrm{pC}$, and the last column indicates the current, but in this study only the second and third column are needed to validate proper operation. The prototype will be shown in Figure 2.

Once the bases of the PDs are known, and how they affect the insulations, we started from there to show measurement methods thereof, between the normal or traditional method and the oscillating; in this article only measurements were taken of the standard method using a measurement type LDS-6 and applying the norm IEC 60270.

Noting Figure 2 the following can be identified: in paragraph (a) it is observed that the insulating material already had a bubble inside causing internal PDs; in paragraph (b) it is the peak effect which generates corona discharge; in paragraph (c) it has PD superficial imperfection because it is worn on the insulation surface; in paragraph (d) false contact was found where a joint was loosened holding to the terminal of the source; in paragraph (e) the same method as in the preceding paragraph was performed but is immersed in transformer oil; and finally in subsection (f) an item on a wooden surface which has not caused potential and is generating a float voltage is placed.

2.1. Databases. Following the most representative measurements of the DPs are shown, to have a better appreciation of the behavior of each of the types.

In Figure 3 it can be observed that internal DPs have a high concentration at the beginning of the cycle in both the positive part and the negative part and a low magnitude.

In Figure 4 the corona discharge that is generated only in the half negative cycle and magnitudes greater than $40 \mathrm{pC}$ can be observed.

The DPs that were generated in an underground cable where several cuts were made to model the measurements that are shown in Figure 5 have the characteristic that the DPs arise both in the positive half cycle and in the negative half cycle and have a great magnitude greater than $100 \mathrm{pC}$.

The false contact can be seen in Figure 6. It has the characteristic of appearing at the beginning and at the end of the semicycles, this being the inverse in magnitude that depends on the position of the cycle.

The primary feature of the corona discharge when immersed in oil is the one which represents PD as positive and negative in the negative half cycle but with a lesser magnitude than in air because the oil reduces the magnitude of the discharge that can be seen in Figure 7. 


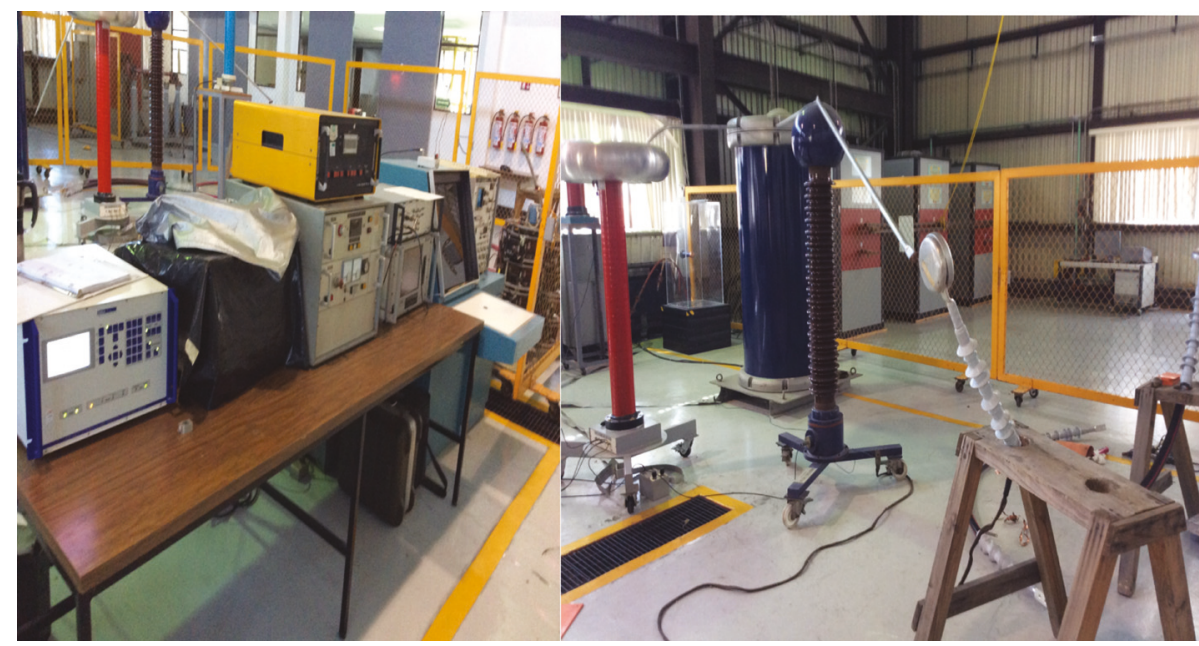

FIgURE 1: Laboratory tests, LAPEM.

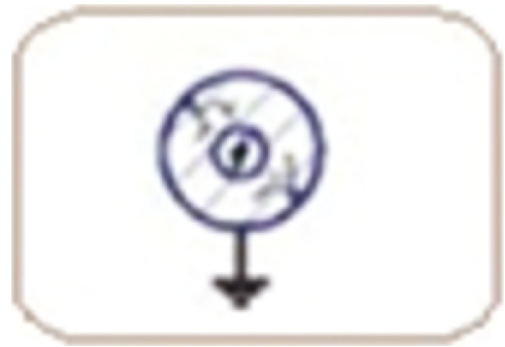

(a) Internal PD

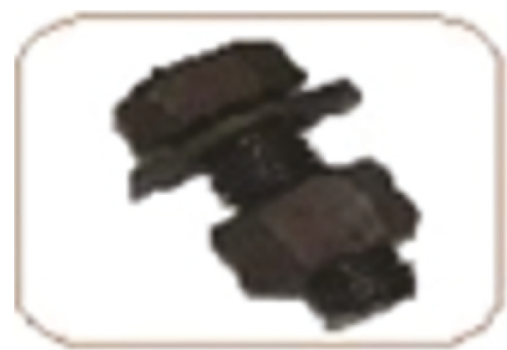

(d) False contact

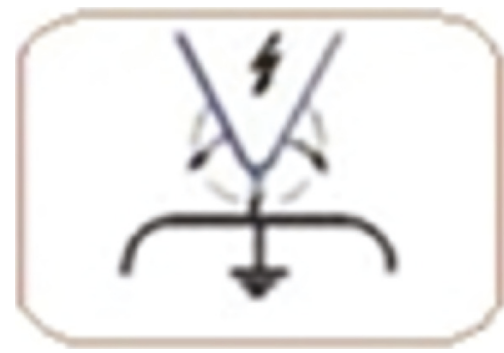

(b) Corona discharge

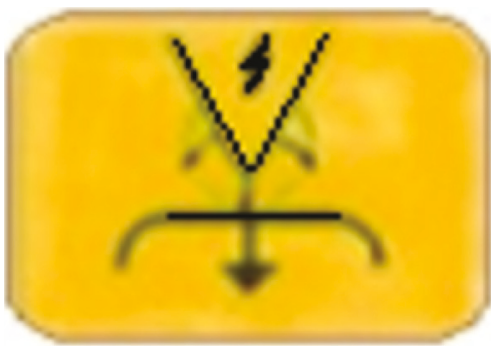

(e) Corona discharge, oil

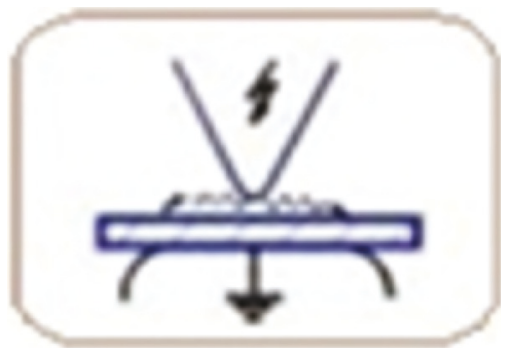

(c) Superficial PD

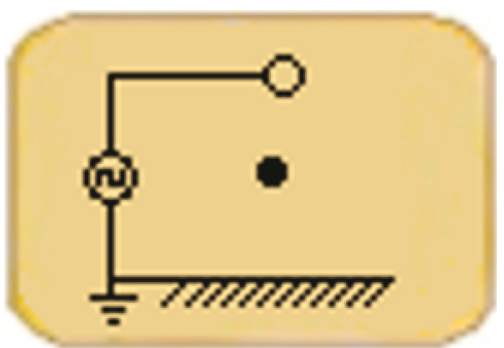

(f) Float voltage

Figure 2: Prototype of different PDs.

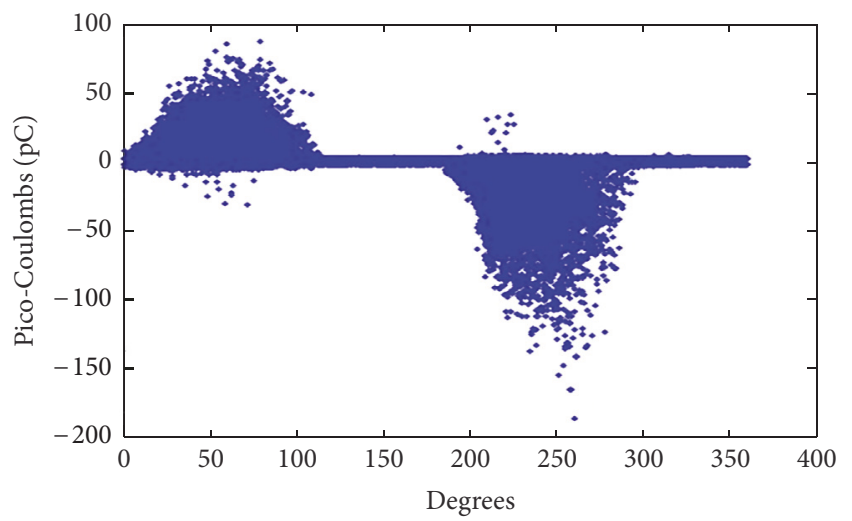

FIGURE 3: Graph of internal partial discharge [16].
The floating ground can be seen in Figure 8; this is only represented at the angles of $200^{\circ}$ to $250^{\circ}$ with a variable magnitude between 300 and $600 \mathrm{pC}$.

\section{Probabilistic Neural Network}

Probabilistic neural networks (PNN) belong to the family of neural networks with radial basis function (String et al., 2008). In 1989 Donald F. Specht published his work "Probabilistic Neural Networks," which introduced the development of a probabilistic neural network (PNN) capable of estimating limits or nonlinear decision surfaces through optimal Bayesian approach. In the case of PDs these characteristics are optimal because they are not complicated because it does not 


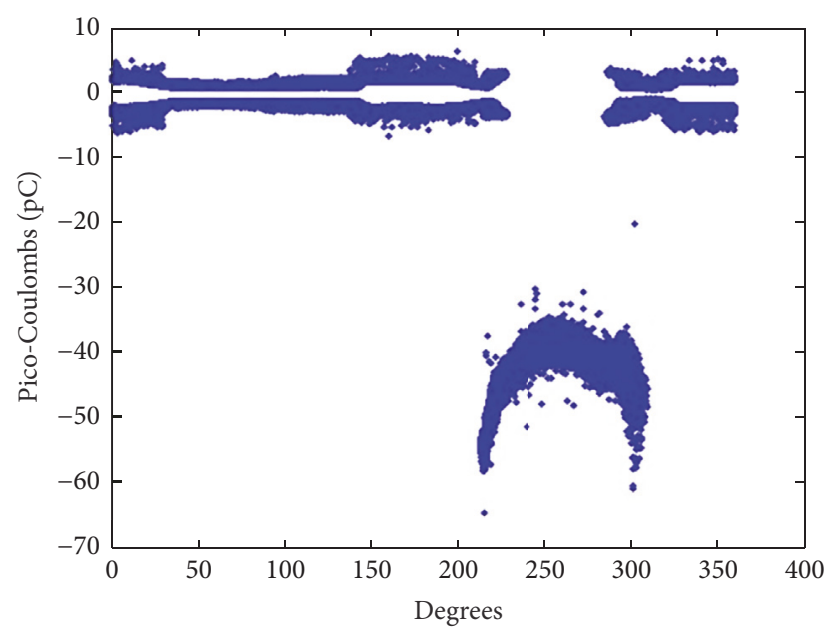

FIGURE 4: Graph of corona in air [16].

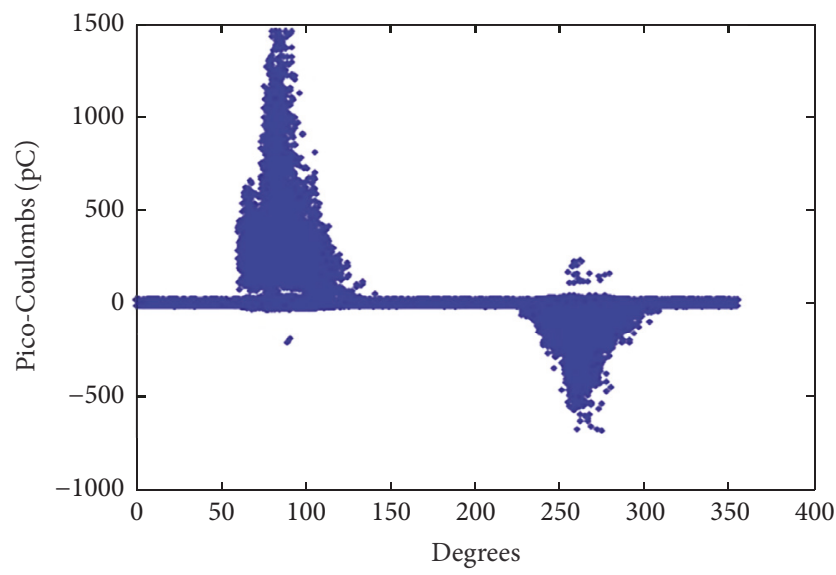

FIGURE 5: Partial discharge on the surface of a XLPE cable [16].

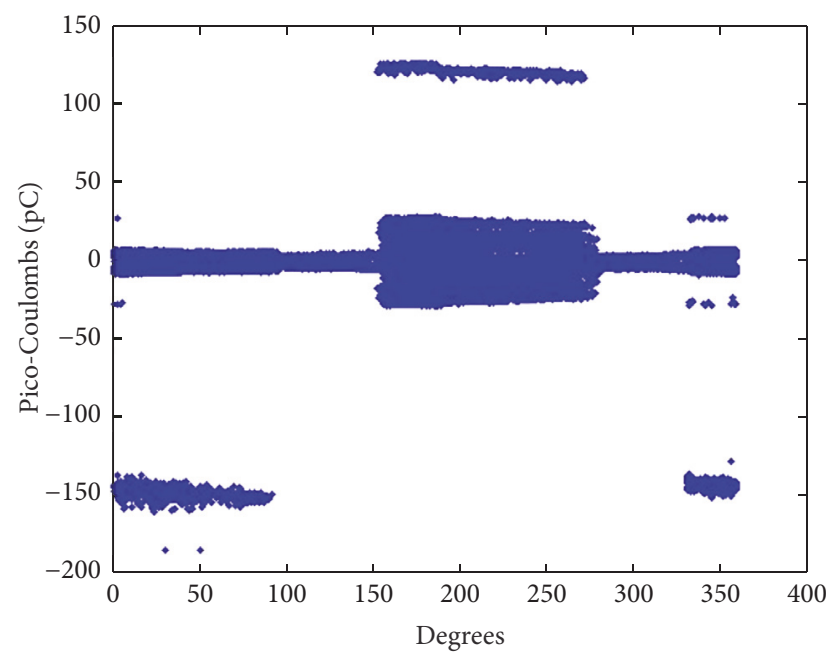

FIgURE 6: False contact of a joint of a XLPE cable.

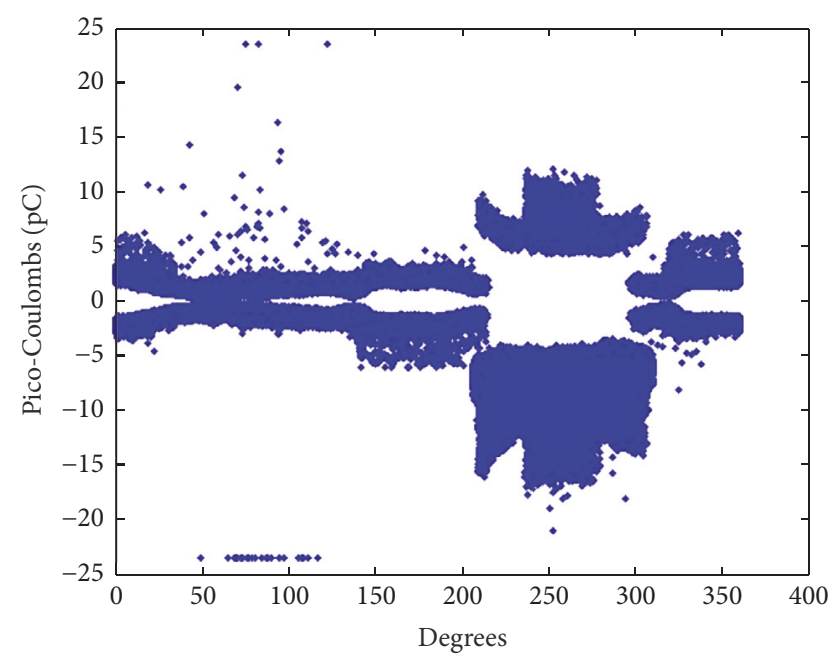

FIGURE 7: Corona discharge immersed in transformer oil.

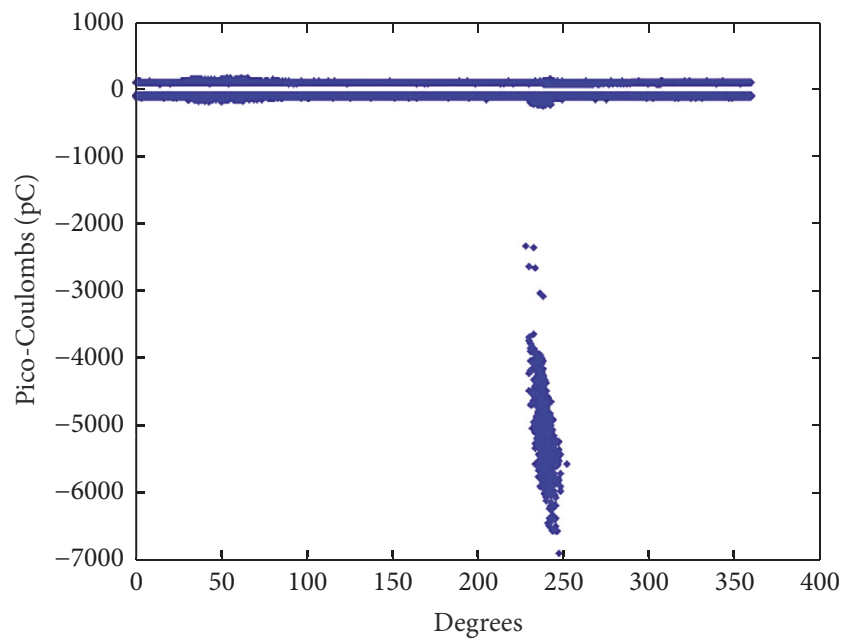

Figure 8: The floating ground.

have to determine intermediate networks and this improves the measurements by the large amount of data that needs to make a measurement in a short time.

3.1. Bayesian Classification Rule. The classification rule optimal Bayesian can be defined as follows, given a collection of random samples of $n$ populations. The a priori probability that the sample $y_{i}$ belongs to the $k$ population is denoted as $h_{k}$. The cost associated with a misclassification that a sample belongs to the $k$ population is denoted by $l_{h}$. The conditional probability that a specific sample belongs to the $k$ population $p\left(k y_{i}\right)$ is given by the probability density function $f_{h}(y)$. Therefore, a sample $y_{i}$ is classified within the $k$ population if it meets the condition established in (1), in the case of two populations.

$$
h_{k} l_{k} f_{k}\left(y_{k}\right)=h_{i} l_{i} f\left(y_{i}\right) .
$$

Contrasting the learning process that takes place in the majority of artificial neural networks, in which an adjustment 


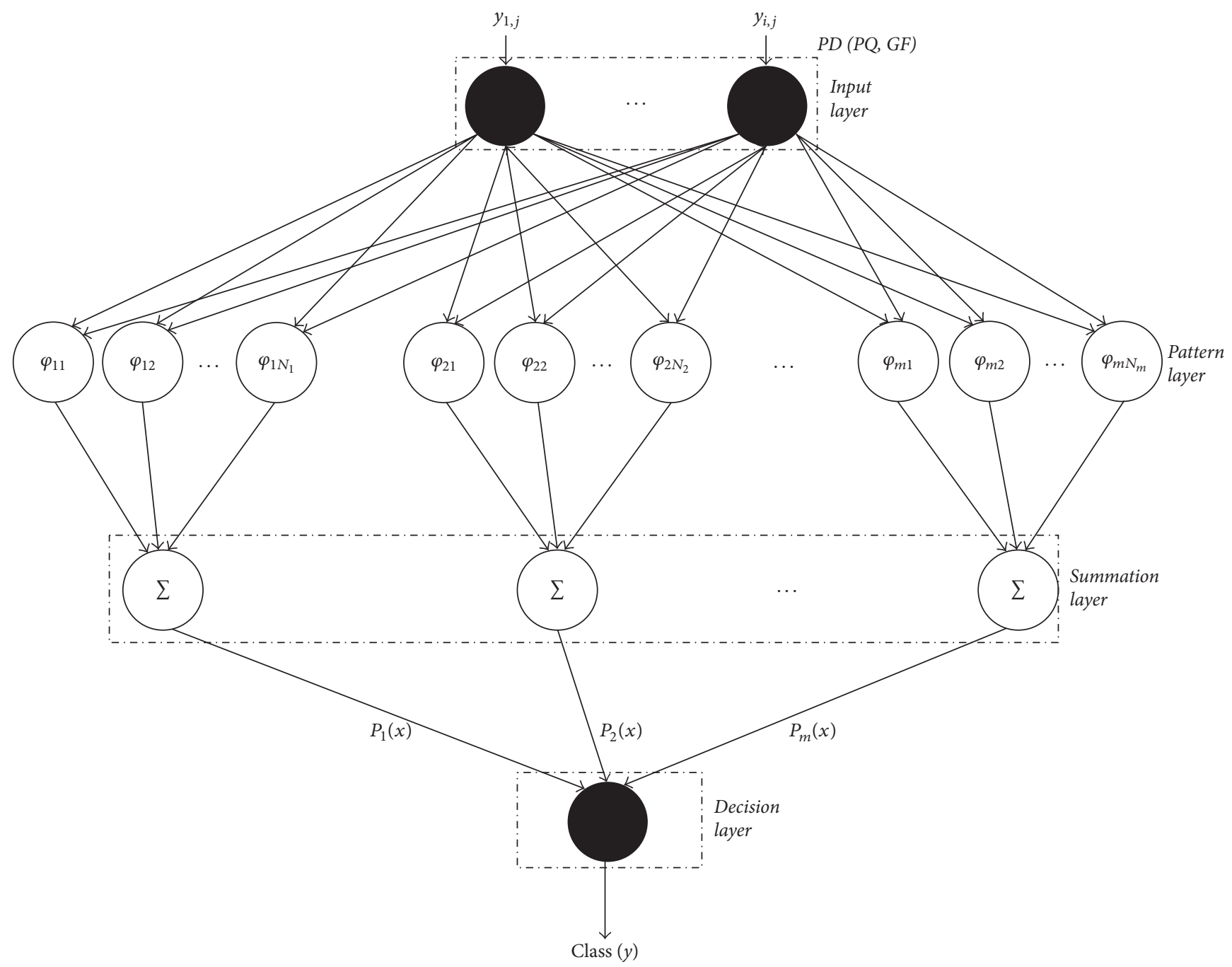

FIGURE 9: Structure of a probabilistic neural network.

to the values of parameters known as weights $\left(y_{1}\right)$ and bias $\left(y_{b}\right)$ is performed, making use of a PNN is not needed to make any adjustment weights and only the output patterns are determined by comparing and calculating the distances of each of the patterns or vectors of input data with each one of the so-called patterns examples, which as their name says are representative examples of the patterns of each of the existing classes.

3.2. Structure of a PNN. Probabilistic neural networks are composed of four layers: an input layer which consists of $d$ neurons where $\varphi_{i j}$ is the dimension of input data, a layer of patterns which consists of $N$ neurons, one for each vector example, a summation layer of $k$ neurons where $k$ is the number of classes, and a layer of decision which is a neuron; the above description is depicted in Figure 9.

When a vector is represented to the network $y=$ $\left[y_{1 j} \cdots y_{i j}\right]$ to be classified, the second layer deals with calculating the distances from the input vector to each of the vectors or patterns, for example, through function $\varphi_{i j}$ which acts according to the standard normal kernel used as the probability density function as shown in

$$
\varphi_{i j}(y)=\frac{1}{(2 \pi)^{d} 2 d^{d}} * \exp \left[\frac{\left(y-x_{i j}\right)^{f}\left(y-x_{i j}\right)}{2 g^{2}}\right] .
$$

In (2), sigma $(\sigma)$ is the dispersion parameter, which takes a value between 0 and 1 to be defined by the researcher. The term $P_{t}(y)$ in the summation layer indicates the conditional probability or verisimilitude that $y$ (input data) belongs to the $i$ th class and is obtained through a process of summation as shown in

$$
P_{i}(y)=\frac{1}{N_{i}} \sum_{j=1}^{N i} \varphi_{i j}(y) .
$$

In the output layer it will be assigned to the class with greater verisimilitude, complying with the provisions of

$$
\text { Class }(y)=\underset{i}{\arg \max }\left(P_{i}(y)\right),
$$

where class $(y)$ is the variety to which $x$ belongs. 


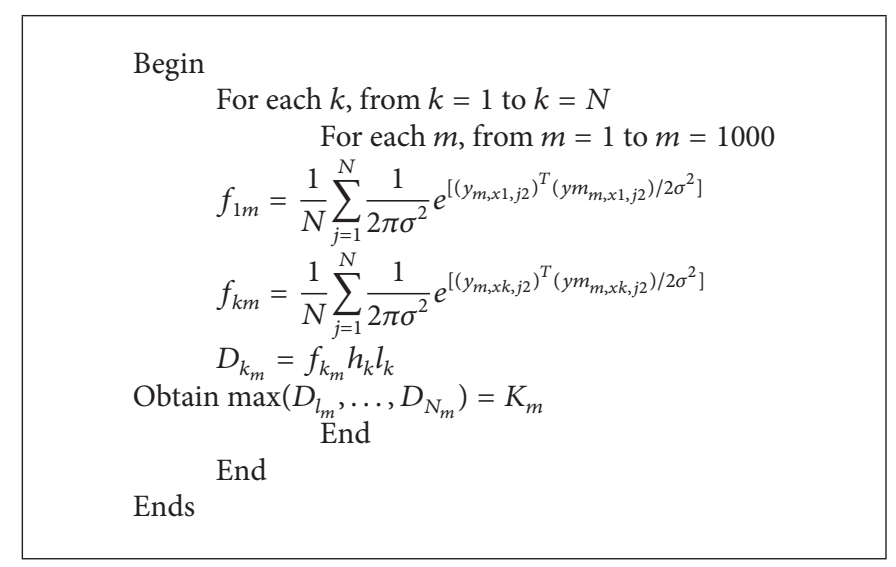

Pseudocode 1

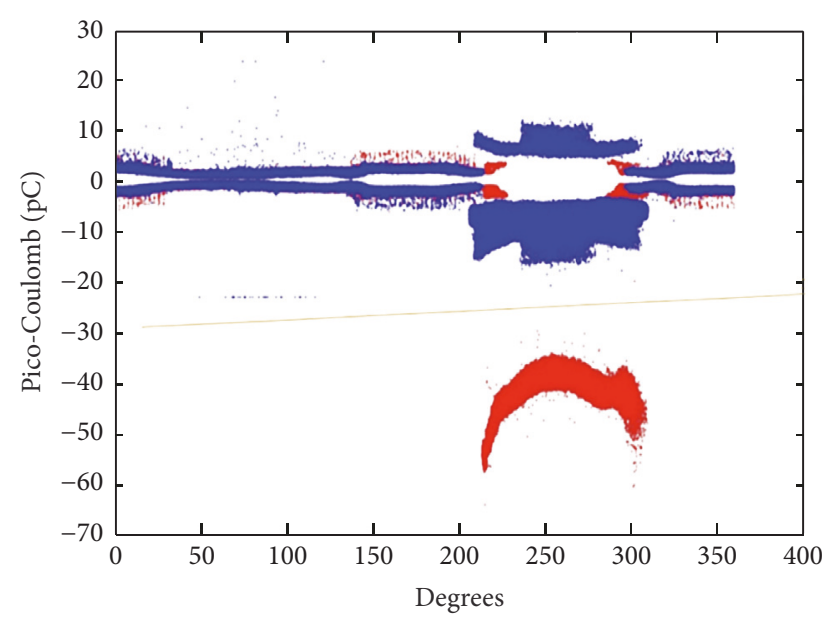

Figure 10: Actual data of partial discharge measurements.

\section{Description of Data under Study}

Data were generated through a simulation process using the MATLAB ${ }^{\circledR}$ normrnd command; the data were generated under the features presented by the actual data, the product of PD measurements, angle, and magnitude. To define a real and proper classification to such data, inferior and superior specifications for both characteristics were considered. Figure 10 shows the inside of PD measurements and corona type and where and how the magnitude is at pico-Coulombs; in this graph the specifications for features that are linearly separable and observed exist.

Figure 11 shows an example of data generated by simulation which were subsequently classified by the PNN to verify the effectiveness of the classification of the network.

4.1. Stage 1: Classification in Six Classes. In the first instance the classification problem considering six classes arises; these classes are formed by segmenting the $x y$ plane shown in Figures 10 and 11 in six areas, formed precisely by cuts between the lines representing the specifications, so class 1 is formed by the internal PDs and corona is below the

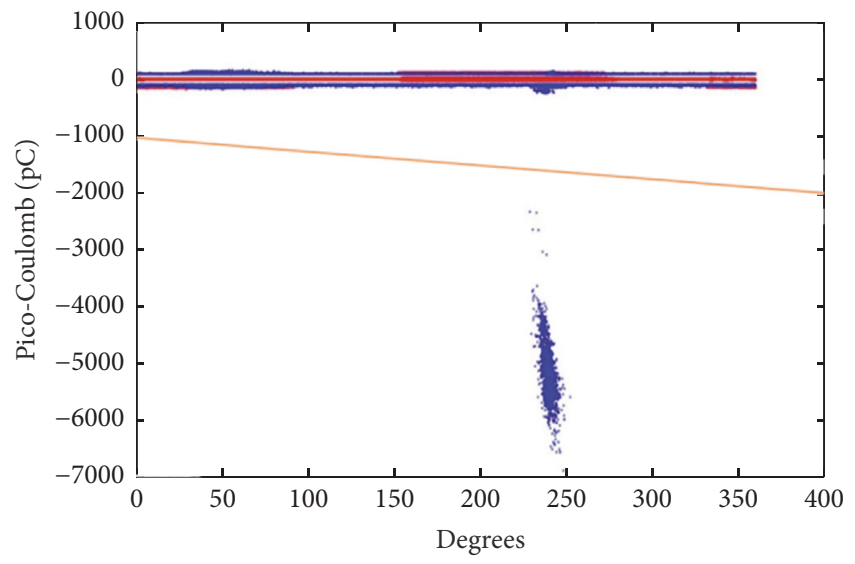

Figure 11: Actual data of PD measurements.

corresponding lower specification; class 2, located to the right of class 1 , corresponds to the superficial PD and false contact $\mathrm{PD}$, the specifications for the characteristic of angle whose magnitude remains lower than established as desirable, and so on.

For application of the PNN with the use of Bayesian classification rule, network simulation in MATLAB software is required; then the pseudocode used for programming is exposed, for which (1), (2), (3), and (4) were conducted in order to identify the effectiveness of network classification to various values of the sigma variable $(\sigma)$ and loss function of class $\left(l_{i}\right)$ in order to find those values that minimize the number of errors during qualifying.

In Pseudocode 1, the matrix $1000 \times 2$ is represented as $y_{m x d}$, which represents the basis of the 1000 data generated from measurements, where each row of said matrix represents a test PD whose first element (first column) indicates measuring the angle and whose second element (second column) indicates the measurement of the magnitude. Similarly to $X_{N x d}$ which is understood as the $N$ patterns example of class $i$, whose elements are denoted by $x_{i j, d}$, there are so many matrices $X_{i}$ as $k$ classes are considered in the problem. 
The $k$ vectors $f_{i_{m x l}}$ are composed of 1000 elements, denoted as $f_{k_{m}}$. The $K_{m}^{m}$ vectors contain 1000 elements whose values range from 1 to $k$, which denotes the class to which the $m$ th data belongs.

For the value of sigma $\sigma$ values were considered: $\sigma=$ $0.2,0.4,0.6,0.8$, and 1 , while for values $l_{i}$ it was specified for researcher that tests will be conducted only modifying the parameter for one of the six classes; class 5 was selected because it corresponds to the class where the measurements data exist, in which they are within specifications for both angles as the magnitude; therefore it would incur greater economic loss by classifying data located in this category incorrectly; the class 5 is a critical category.

The values are considered for $l_{s}$ were $0.11,0.12,0.13,0.14$, $0.15,0.16$, and 0.17 . For each value of $l_{s}$ considered, loss values for the other classes were defined, following the rule that when the value increased, the loss value for the other classes would decrease in the same proportion for all, thus maintaining the equalities shown in (5) and (6) during the experimentation.

$$
l-l_{s}=l_{1}+l_{2}+l_{3}+l_{4}+l_{5}+l_{6}+l_{7}+l_{8}+l_{9}
$$

where

$$
l_{1}=l_{2}=l_{3}=l_{4}=l_{5}=l_{6}=l_{7}=l_{8}=l_{9} .
$$

With the results of the previous experiments, it is specified that the best values for the parameters of the network are $\sigma=0.2 h_{t}=l / 9$ y $l_{t}=l / 9$, where the subscript $i$ indicates the class which is referred, where $i=1,2,3,4,5,6$ for the first stage of classification problem.

With the previously defined values, PNN was applied again in 35 databases containing 1000 data samples each, in order to obtain an average of the number of errors and proportion of successes presented in this network for classification in six categories.

A level of acceptance for the resolution of the classification problem is established prior to the investigation, if the network has an efficiency greater to $90 \%$. For the first stage of the problem presented, the desired result was not obtained. Several tests were performed increasing the number of patterns to check if this contributed to achieving the objective raised with several examples; however, as no satisfactory results were obtained, a second stage to solve the classification problem was established.

4.2. Stage 2: Classification in Two Classes. For the second stage to resolve the classification problem set out the reduction of the number of classes which is categorized in the desired data, following the same pseudocode used in the previous stage; this reduction in the number of classes was carried out considering that, in the literature on the application of PNN, experimental tests have been for rating only two classes and they all had a high rate of effectiveness in terms of number of successes made by the network.

The problem of data classification with a focus on categorization in two classes was considered. This arises when attention of the problem is focused on identifying those data found within all specifications (class located at the center of Figure 11) as elements of class one and the data that do not know one or more of the specifications as elements of a second class.

The established values for this new approach to the classification problem were

$$
\begin{aligned}
g & =0.2, \\
h_{1} & =\frac{l}{g}, \\
h_{2} & =\frac{2}{g}, \\
l_{t} & =\frac{l}{2},
\end{aligned}
$$

where $i=1$ is the identifier for class one which contains the data found within all specifications; the value of $h_{1}$ was established considering that only one-ninth of the population data will be within all specifications as noted in the test characteristics and the rest of the population will be out of at least one of the specifications; this is observed from Figures 10 and 11. With this new approach to PNN, high levels of successes in the classification of data were obtained.

4.3. Stage 1: Training of Six Classes. Probabilistic neural networks (PNN) can be used for data classification problems.

The process used for regression problems is one in which adjustment of weights and bias is performed, according to the presented error; in the classification process no adjustment is made of weights and only the output patterns are determined by comparison and distance calculation. In Figure 12 the network configuration is presented.

Its operation can be explained as follows: when an input is presented to the network, the first layer is responsible for calculating the distances from the input vector to the output vectors and produces a vector whose elements indicate how close is the entrance with respect to the output. The function of the second layer is to add the contributions for each class of input and produce with them an output vector with probabilities.

Finally, a transfer function at the output of the second layer of "competitive" type, select the maximum of these probabilities and produces 1 for that class and 0 for others.

\section{Network Configuration to Classify the Different PD Patterns}

The application of neural networks in the fault diagnostics has two stages. Stage one corresponds to the training process, during which the training patterns are supplied to the network in order to perform calculations or adjust some of its parameters. Stage two is the testing process, during which an unknown pattern data is delivered, in order to verify whether the output delivered by the network corresponds to the expected output.

Some simulations were performed to determine the extraction patterns of the PNN; the data of patterns extraction 
Network architecture

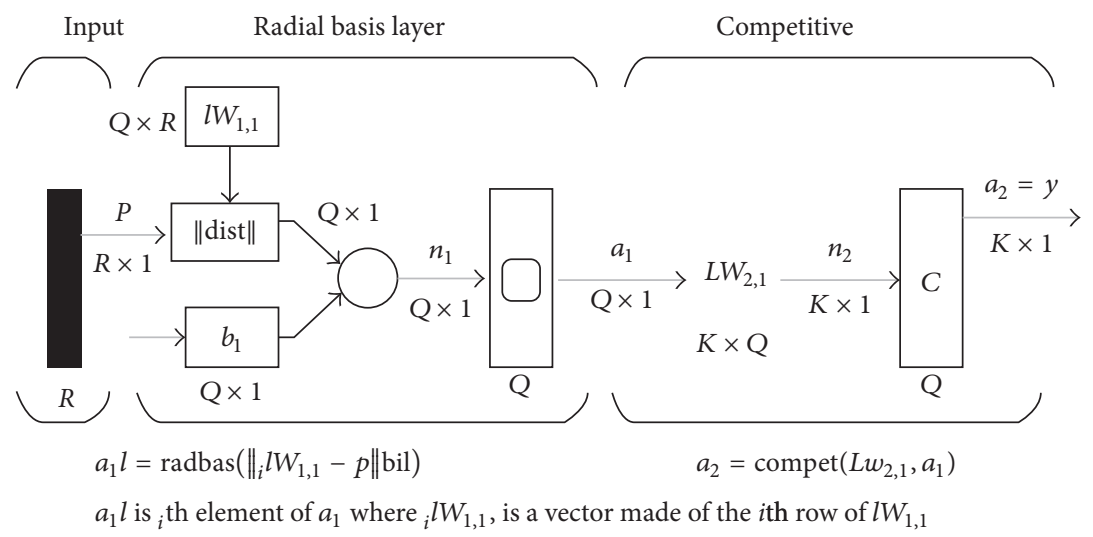

Where...

$R=$ number of elements in input vector

$Q=$ number of input/target pairs = number of neurons in layer 1

$K=$ number of classes of input data $=$ number of neurons in layer 2

FIGURE 12: Architecture of a probabilistic neural network [17].

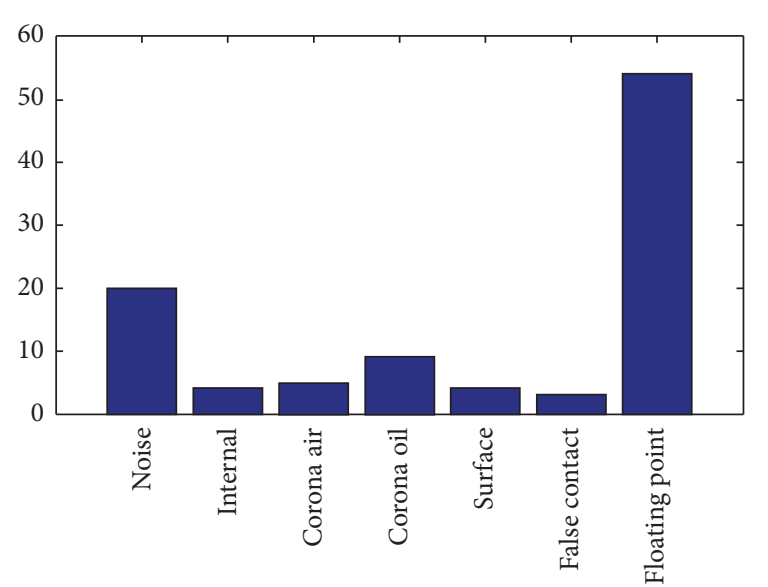

FIGURE 13: Results are presented as percentages and indicate internal partial discharge.

was determined in a percentage way as shown in Figure 13; this is in order to see clearly the data concentration.

Finally, it proceeded to validate the network, and data vector containing the class type and amount of data from each of these was obtained, but to have a better appreciation of these data, in Figure 13 percentage form the data obtained is presented; with the figure it is very easy to determine the type of class.

In this case it was validated with a measurement where it was known that the PD was internal and the results are shown as follows: the first column presents the class where the data that are noise exist, in the column seven are the internal PD, in six (6) there are the external discharges in air, in column five (5) there is floating ground, in four (4) there is corona in air, in column three (3) there is the corona discharge in air, and in two (2) there is the floating ground; it can be seen clearly in Figure 13 that it is internal PD due to the high intensity that has column seven which generates the data to be very easy to determine.

\section{Study Cases}

In this section the following case studies will be carried out to analyze the quality of insulation of XLPE high voltage cables for a nominal voltage of $115 \mathrm{KV}$, where two types of cable producers from different manufacturers were used.

The test was made up of

(1) using 5 meters of cable made of a copper cable and insulated by a cross-linked polymer (XLPE), with semiconductor shield that was extruded over the insulation and metal screen covered with polyvinyl chloride (PVC);

(2) PDs measurements made with the LDIC equipment using a $75 \mathrm{kV}$ considering the parameters of the IEC 60270 norm as shown in Figure 1;

(3) acquisitions of the measurements that were recorded in a computer system with LDIC software;

(4) Simulation of the PNNBM.

The results of the measurements and the simulations of the PNNBM are described in detail as follows.

6.1. Cable 1. (1) The following percentages of PD patterns were obtained for manufacturing cable \# 1 as shown in Figure 14. The results of the PNNBM simulation show $77.8 \%$ of internal $\mathrm{PD}$; this indicates that the internal insulation which covers the main cable has a deficiency and may have a propensity to insulation failures in a short period of time. It can also be observed that the external insulation of the cable presents a small degree of degradation since it presents $3.75 \%$ of superficial PD. The results show that it is necessary for the manufacture of cable insulation to be of higher quality 


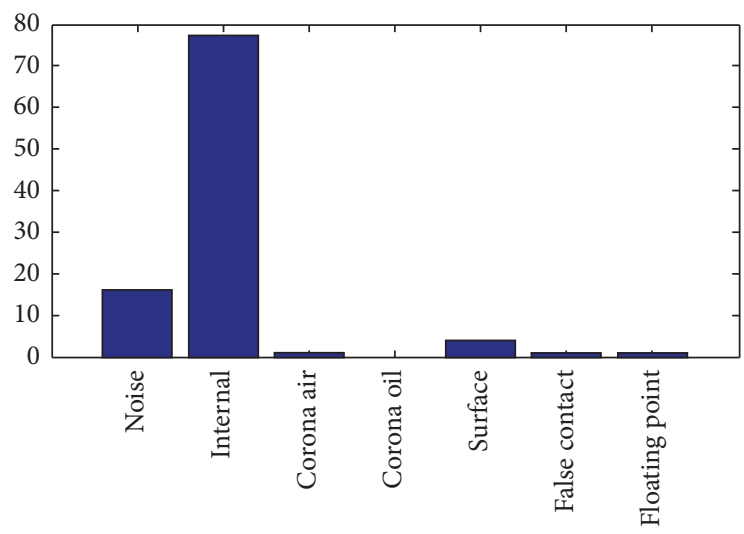

FIgURE 14: Actual measurement of the first cable.

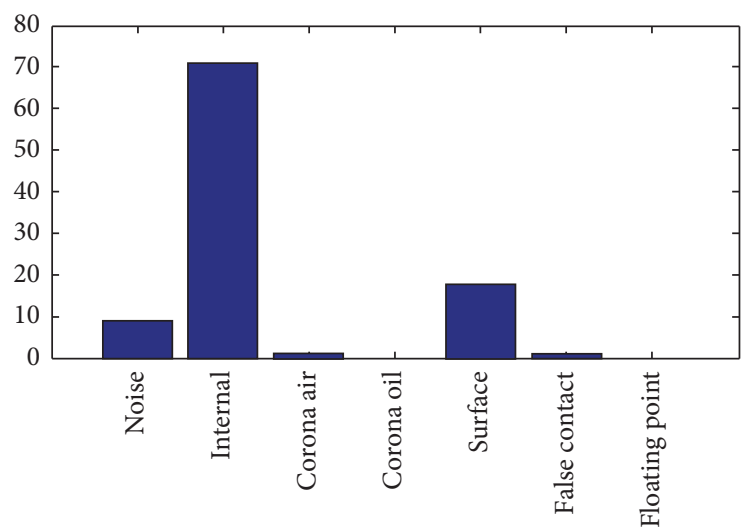

FIGURE 15: Actual measurement of the second cable.

because of the electrical stresses that will be subjected to the high voltages.

On the other hand, the cable also presents a small proportion of PDs of $1.2 \%$ by corona effect related to the terminals derived in tips. They present $1.3 \%$ and $1.23 \%$ of PDs of false contact and floating point, respectively. These three types of DPs are mainly related to the connection and installation of the cable.

6.2. Cable 2. Finally, the second cable was measured using the same characteristics mentioned above. Previously trained, the neural network PNNBM proceeds to the simulation that gives results as those of Figure 15.

It can be estimated in Figure 15 that the cable has a concentration of $70.28 \%$ of internal PD, this being a new cable, and has a considerable presence of $18.53 \%$ of superficial PD; the other types of PDs are discarded because they are not relevant to analysis for their magnitudes are less than $1 \%$; due to these results this cable should no longer be installed.

6.3. Comparison. To compare the results of the neural network, a statistical method for measurements of PDs of both wires, resulting Table 1 was used, verifying the obtained results of the neural network which can be seen to effectively have a high concentration of internal PD; the statistical
TABLE 1: Statistical parameters of two different cables.

\begin{tabular}{ccccccc}
\hline & $\mu$ & $\sigma^{2}$ & $S_{k}$ & $K_{u}$ & $D_{a}$ & $C_{c}$ \\
\hline CABLE_1 & 2.5442 & 27.1492 & 3.4944 & 16.5593 & 2.3774 & -0.2262 \\
CABLE_2 & 4.4018 & 63.9289 & 2.5774 & 9.2703 & 4.5777 & -0.3473 \\
\hline
\end{tabular}

parameters of the formulations presented in Table 1 can be found in reference [18].

Table 1 establishes the variance average of $2 \mathrm{pCs}$ implied that refers to PD Internal by their small magnitude; in the part of the standard deviation, a considerable differentiation of dimension is observed, establishing that there is a large scatter in the data of cable two, resulting in two different patterns; the bias does not vary with respect to cables unlike kurtosis in cable 1 which is almost double cable two; this tells us that the concentration of PD in the cable 1 is greater, which determines that cable 1 is prone to generate a fault. In the part of the correlation factor it indicates that naturally it comes to the same types, that is why these are negative values not greater than the least one.

\section{Conclusions}

This paper proposed a prototype that manages to classify the 4 classic patterns (internal PD, superficial PD, corona discharge in air, and corona discharge in oil) and 2 new patterns proposed (false contact and floating ground) of $\mathrm{PD}$ in the insulation of high voltage cables, XLPE. To classify the types of PD a new neural network (NN) is implemented which was trained to get better results and reduced errors. The results showed that the proposed PNBM was not saturated with large volumes of information and always converges, finding the solution. PNBM was implemented in two cables with similar features from different manufacturers. The results showed that the two cables present internal PD; however one of them has high concentrations of superficial PD which indicates that insulation is of poor quality.

\section{Conflicts of Interest}

The authors declare that there are no conflicts of interest regarding the publication of this paper.

\section{References}

[1] A. Rodrigo Mor, L. C. Castro Heredia, and F. A. Muñoz, "Effect of acquisition parameters on equivalent time and equivalent bandwidth algorithms for partial discharge clustering," International Journal of Electrical Power \& Energy Systems, vol. 88, pp. 141-149, 2017.

[2] R. Jia, Y. Xie, H. Wu, J. Dang, and K. Dong, "Power transformer partial discharge fault diagnosis based on multidimensional feature region," Mathematical Problems in Engineering, vol. 2016, Article ID 4835694, 11 pages, 2016.

[3] M. E. Ibrahim and A. M. Abd-Elhady, "Calculation of electric field and partial discharge activity reduction for covered conductor/high voltage insulator systems," Electric Power Systems Research, vol. 144, pp. 72-80, 2017. 
[4] M. Majidi and M. Oskuoee, "Improving pattern recognition accuracy of partial discharges by new data preprocessing methods," Electric Power Systems Research, vol. 119, pp. 100-110, 2015.

[5] A. A. Bajwa, "An investigation into partial discharge pulse extraction methods," International Journal of Electrical Power \& Energy Systems, vol. 73, pp. 964-982, 2015.

[6] K. Dieter, High-Voltage Insulation Technology: Textbook for Electrical Engineers, High-Voltage Insulation Technology, Braunschweig, Germany, 2015.

[7] S. A. Boggs, "Partial discharge: overview and signal generation," IEEE Electrical Insulation Magazine, vol. 6, no. 4, pp. 33-39, 1990.

[8] W. J. K. Raymond, H. A. Illias, A. H. A. Bakar, and H. Mokhlis, "Partial discharge classifications: review of recent progress," Measurement, vol. 68, pp. 164-181, 2015.

[9] S. Venkatesh and S. Robust, "Heteroscedastic probabilistic neural network for multiple source partial discharge pattern recognition - significance of outliers on classification capability," Expert Systems with Applications: An International Journal archive, vol. 38, no. 9, pp. 11501-11514, 2015.

[10] M.-S. Su, "Classification of partial discharge events in GILBS using probabilistic neural networks and the fuzzy c-means clustering approach," International Journal of Electrical Power and Energy Systems, vol. 61, pp. 173-179, 2014.

[11] A. Abubakar Mas'Ud, B. G. Stewart, and S. G. McMeekin, "Application of an ensemble neural network for classifying partial discharge patterns," Electric Power Systems Research, vol. 110, pp. 154-162, 2014.

[12] O. Perpiñan, "Signal analysis and feature geration," Electric Power Systems Research, vol. 95, pp. 56-65, 2013.

[13] J. Granado, C. Álvarez-Arroyo, A. Torralba, J. A. RosendoMacías, J. Chávez, and M. Burgos-Payán, “Time domain analysis of partial discharges envelope in medium voltage XLPE cables," Electric Power Systems Research, vol. 125, pp. 220-227, 2015.

[14] F.-C. Gu, H.-C. Chang, F.-H. Chen, and C.-C. Kuo, "Partial discharge pattern recognition of power cable joints using extension method with fractal feature enhancement," Expert Systems with Applications, vol. 39, no. 3, pp. 2804-2812, 2012.

[15] T. Hang, J. Glaum, T. Phung, and M. Hoffman, "Investigation of partial discharge and fracture strength in piezoelectric ceramics," Journal of the American Ceramic Society, vol. 97, no. 6, pp. 1905-1911, 2014.

[16] F. F. Godoy, J. M. G. Guzmán, R. J. Vacio, and F. J. O. Herrera, "Obtaining measurement patterns of partial discharges in power cables XLPE using Probabilistic Neural Networks," International Journal of Scientific and Research Publications, vol. 5, no. 3, 2015.

[17] A. Hekmati, "A novel acoustic method of partial discharge allocation considering structure-borne waves," International Journal of Electrical Power \& Energy Systems, vol. 77, pp. 250255, 2016.

[18] M. Heidari, "Combined Diagnosis of PD Based on the Multidimensional Parameters," Modelling and Simulation in Engineering, vol. 2016, Article ID 5949140, 12 pages, 2016. 


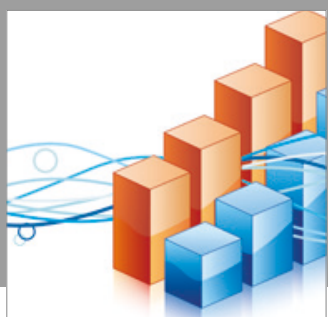

Advances in

Operations Research

vatersals

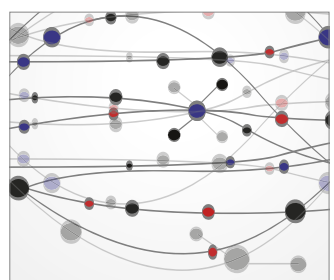

\section{The Scientific} World Journal
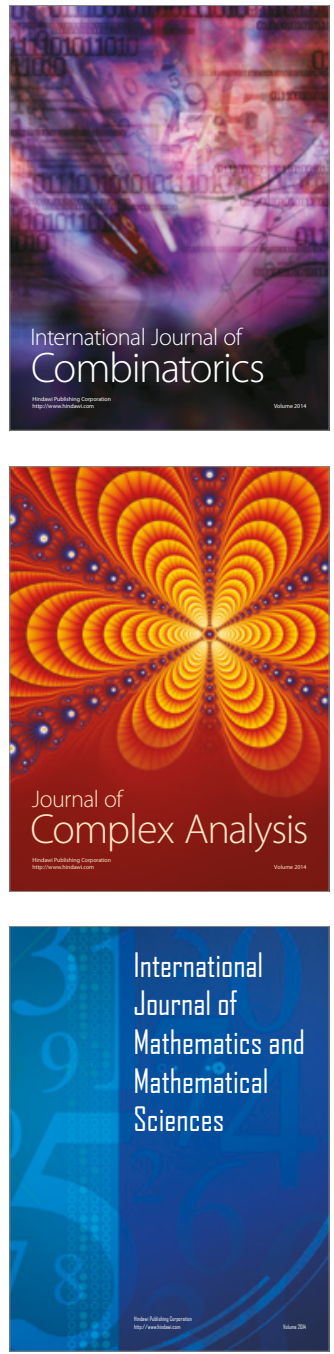
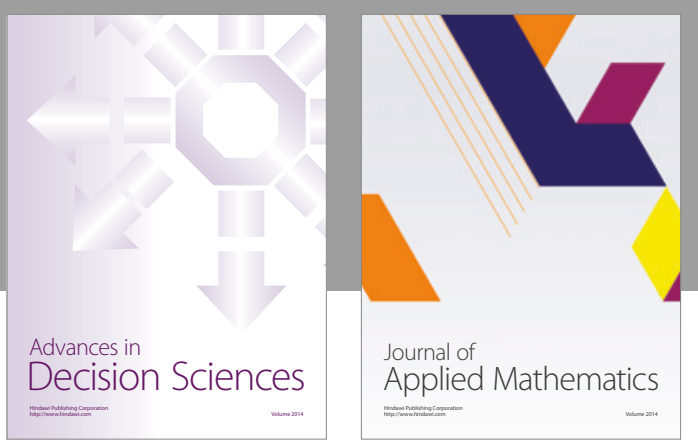

Algebra

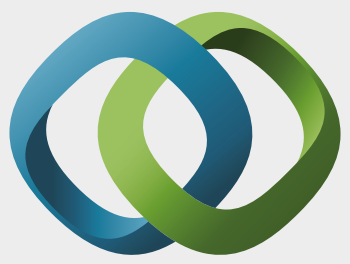

\section{Hindawi}

Submit your manuscripts at

https://www.hindawi.com
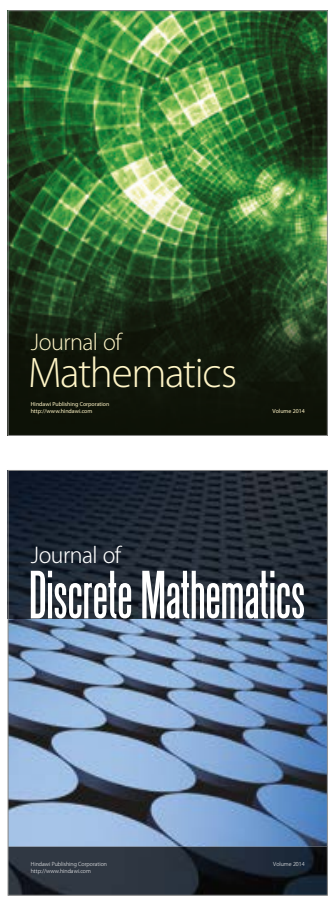

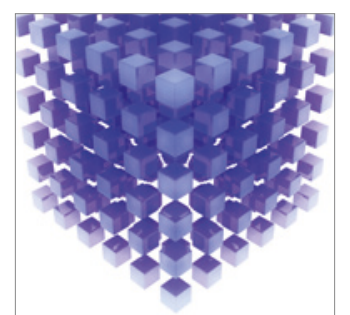

Mathematical Problems in Engineering
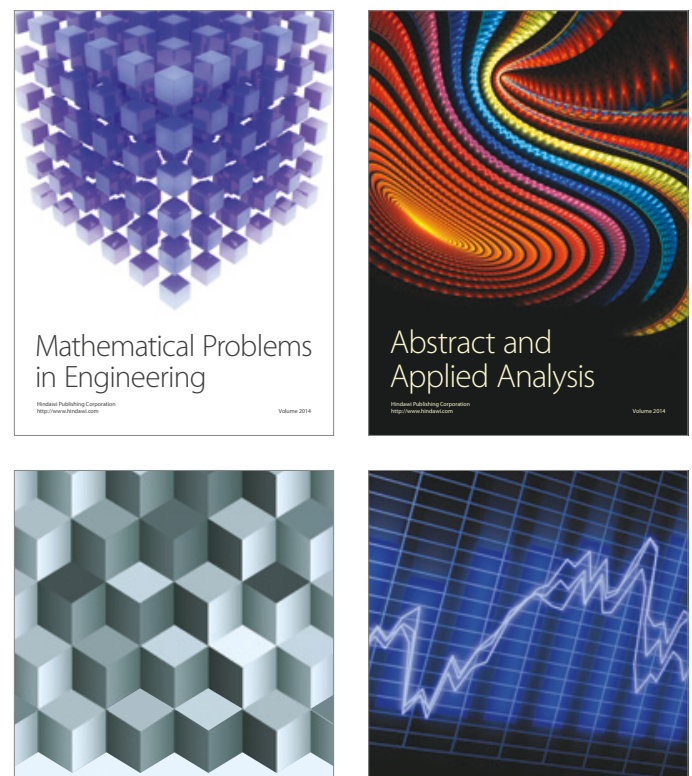

Journal of

Function Spaces

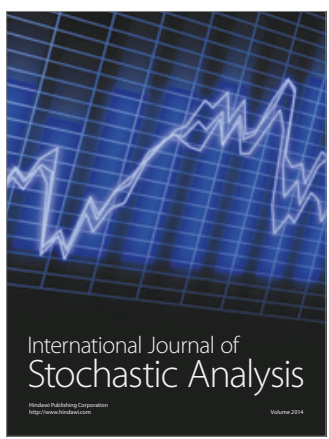

Probability and Statistics
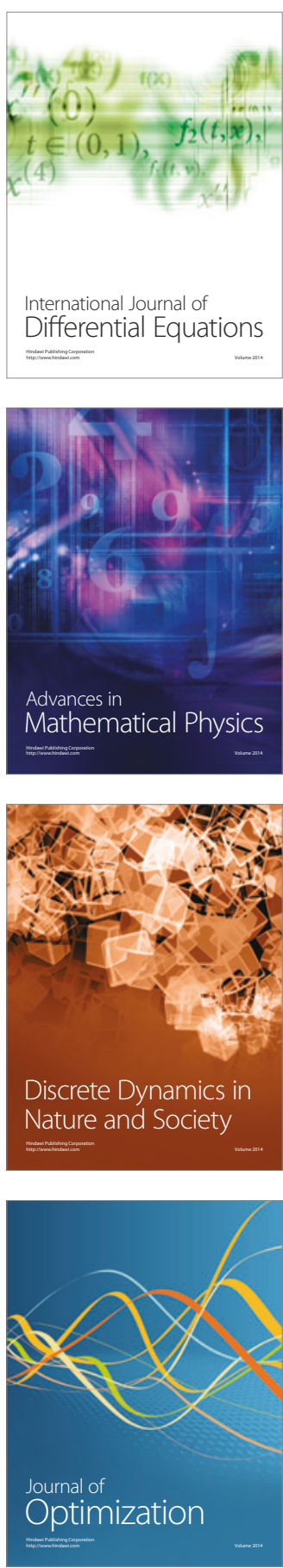